\title{
Clinical Effects of an ACT-Group Training in Children and Adolescents with Attention-Deficit/Hyperactivity Disorder
}

\author{
Laura Vanzin ${ }^{1}$ - Valentina Mauri ${ }^{1} \cdot$ Angela Valli $^{1}$ Marco Pozzi ${ }^{2} \cdot$ Giovambattista Presti $^{3} \cdot$ Annalisa Oppo $^{4}$. \\ Arianna Ristallo ${ }^{5} \cdot$ Massimo Molteni $^{1} \cdot$ Maria Nobile $^{1}$
}

Published online: 11 September 2019

(C) The Author(s) 2019

\begin{abstract}
Objective The aim of the present study is evaluate the effectiveness of an Acceptance and Commitment Therapy (ACT)based training protocol, in adjunct to token economy and previous parent training, in a sample of children with AttentionDeficit/Hyperactivity Disorder (ADHD). By promoting the reduction of immediate responses to thoughts and feelings, we aimed to reduce the impulsive behaviour of children and to improve their self-regulation.

Methods The protocol was centred on awareness of the present moment, defusion and acceptance of feelings and emotions. Behavioural (Conners' Parent Rating Scale -Revised: Long version, CPRS-R:L) and severity measures (Clinical Global Impression -Severity, CGI-S) were assessed before and after treatment in a clinical sample of 31 children aged 8-13 years. Results At the end of the ACT protocol, children showed significant improvement in global functioning and behavioural symptoms. There were significant improvements in the CPRS subscales Cognitive Problems $(p=0.005)$, Hyperactivity $(p=0.006)$, Perfectionism $(p=0.017)$, ADHD Index $(p=0.023)$, Global Index: Restless-Impulsive $(p=0.023)$, Global Index: Total $(p=0.036)$, DSM IV Inattentive $(p=0.029)$, DSM IV Hyperactive-Impulsive $(p=0.016)$, and DSM IV Total $(p=0.003)$. When controlling for the confounding effect of pharmacological therapy, comorbidities and socio-economic status, treatment maintained a significant effect on the CPRS subscales Perfectionism (partial $\eta^{2}=0.31, p<0.01$ ), Global Index: Restless-Impulsive (partial $\eta^{2}=0.29, p<0.01$ ), Global Index: Total (partial $\eta^{2}=0.31, p<0.01$ ), DSM IV Hyperactive-Impulsive (partial $\eta^{2}=0.20$, $p=0.02$ ). Symptom severity as rated by CGI-S scores decreased in $74.2 \%$ of the children.

Conclusions This preliminary work on an Acceptance and Commitment Therapy-based child training in children affected by ADHD resulted in significant improvements, measured by a rating scale specific for ADHD.
\end{abstract}

Keywords ADHD $\cdot$ Acceptance and Commitment Therapy $\cdot$ Rehabilitation $\cdot$ Conners parent rating scale $\cdot$ Child training

Attention-Deficit/Hyperactivity Disorder (ADHD) is a neurobehavioral disorder common in childhood, with symptoms including troubles in paying and maintaining

Marco Pozzi

marco.pozzi@lanostrafamiglia.it

1 Child Psychopathology Unit, Scientific Institute, IRCCS Eugenio Medea, Bosisio Parini, Lecco, Italy

2 Scientific Institute, IRCCS Eugenio Medea, Bosisio Parini, Lecco, Italy

3 Faculty of Human and Social Sciences, Kore University, Enna, Italy

4 Sigmund Freud University, Milano, Italy

5 Istituto Europeo per lo Studio del Comportamento Umano (IESCUM), Milano, Italy attention, hyperactivity and impulsive behaviour. The worldwide prevalence of ADHD is estimated at 7.2\% (Thomas et al. 2015), making it the most prevalent psychiatric disorder of childhood. In Italy, prevalence rates range from 1.3 to $7.0 \%$ (Bianchini et al. 2013; Donfrancesco et al. 2014; Gallucci et al. 1993; Mugnaini et al. 2006; Zuddas et al. 2006). ADHD is more frequently diagnosed in boys than in girls, with an estimated ratio of 10:1 in clinic-referred or $3: 1$ in community samples (Biederman et al. 2002). The life domains that are mostly impaired in individuals with ADHD are the academic, occupational, social, mental-health and self-conceptual ones, negatively impacting the lives of children and of their families (Barkley et al. 2008; Matheson et al. 2013; Stein, 2008). ADHD is described as a self-regulation deficit (Barkley 1997; Puiu et al. 2018; Shiels and Hawk Jr 2010). Core symptoms are associated with problems in maintaining 
goals and plans, and an insufficient ability to inhibit impulsive responses (Sharma and Couture 2014). Difficulty focusing attention leads to failure to complete tasks, as the inability to maintain and shift attention does not allow children to foresee what is going to happen in the context. Children often fail to recognize the effect of their behaviour, because of a deficit in their ability to regulate alertness, sustain effort and process information at an appropriate rate (Castellanos and Tannock 2002). From a dynamic behavioural developmental perspective (Sagvolden et al. 2005), ADHD might be explained by a short and steep delay-ofreinforcement gradient, which alongside impaired extinction causes impulsiveness and hyperactivity and hampers the establishment of stimulus control. In this way, individuals with ADHD have difficulties in learning to relate behaviour to its consequences.

Mindfulness is a practice that improves the selfregulation of attention and emotions (Teasdale et al. 1995). It has been described as "a particular way of paying attention on purpose, in the present moment, and nonjudgmentally" (Kabat-Zinn 1994) that helps clients to notice thoughts, sensations, emotions and urges, accepting them without reacting. Mindfulness practice can help training individuals to pay attention to the relevant aspects of their inner and external environment, helping them to notice and learn the relations between events and behaviour (Shapiro et al. 2006). As self-regulation impairment is a key feature of ADHD (Barkley 1997), studies on mindfulness practice have demonstrated its many potential positive effects, including the reduction of behavioural symptoms of inattention and impulsivity, as well as of stress, anxiety and depression (Zylowska et al. 2008). Two recent reviews (Burke 2010; Swain et al. 2015) have found that mindfulness-based interventions are effective for the treatment of children and adolescents with different mental disorders, such as oppositional-defiant and conduct disorder, anxiety, depression, obsessive-compulsive disorder, chronic pain and substance abuse. To our knowledge, there are only a few studies that explore the effects of mindfulness training in children or adolescents with ADHD (Semple et al. 2005, 2010; Van de Weijer-Bergsma et al. 2012; Van der Oord et al. 2012; Zylowska et al. 2008), indicating that mindfulness meditation training is feasible and has positive effects. Mindfulness is a core component of many CBT interventions. Cognitive-behavioural-based programs (i.e. school-based behavioural reinforcement, behavioural parent training and social skills training programmes) are currently among the top evidence-based interventions for treating children with ADHD (The Multimodal Treatment Study of Children with ADHD [MTA] Cooperative Group 1999). However, these programs, which have traditionally focused on putting children's behaviour under the control of an external rule, given by a trainer in a specific context, show several limitations (Van der Oord et al. 2012). We hypothesise that rule-following may reduce the possibility that children directly learn and be regulated by contact with environmental contingencies. This could also explain the well-known lack of generalization to other contexts and behavioural topographies, which different authors have described regarding traditional Cognitive Behavioural treatments for ADHD (Chambles and Ollendick 2001; Pelham and Fabiano 2008).

Murrell et al. (2015) studied the efficacy of an Acceptance and Commitment Therapy (ACT)-based protocol, a so-called 'third wave' cognitive-behavioural intervention (Hayes et al. 2004, 1999; Wilson and Luciano 2002) for the treatment of ADHD. This preliminary investigation assesses the feasibility of using ACT in school-aged children with comorbid ADHD, learning disorders and behaviour problems, in order to increase committed actions connected to personal values. Results suggested that benefits are evident for some children after a short intervention period.

ACT assumes a contextualistic philosophy of science, a theory of language and cognition named Relational Frame Theory (RFT) (Hayes et al. 2001) and an applied theory of psychopathology and psychological change (Hayes et al. 2006). This cognitive-behavioural therapy aims to help clients stuck in their daily lives to pursue a "valued" life, by clarifying personal values and identifying their own goals and actions linked to their values. An ACT protocol aims to build psychological flexibility by encouraging people to be aware of inner experiences and external signals and to stay in contact with the uncomfortable ones, as long as this is what patients consider valued living (Hayes et al. 2006; Simons and Gaher 2005). Several studies show that ACTbased interventions are effective in a number of different clinical and non-clinical contexts, including psychiatric and psychological conditions (such as depression, anxiety, obsessive-compulsive disorders, borderline personality disorder and drug dependence), prevention and health promotion (such as the management of chronic pain, smoking cessation and obesity) and organizational environments (for example, stress management, staff training for teachers, working reintegration, stigma and prejudice) (Öst, 2014; A-Tjak et al. 2015; Ruiz 2010; Powers et al. 2009; Hayes et al. 2006). In recent years, ACT-based interventions have been studied also for patients in the developmental age and some intervention protocols have been applied with children and adolescents (Ciarrochi et al. 2012; Greco and Hayes 2008). Current studies, although pivotal, demonstrated promising effects on chronic pain and psychological disorders (Swain et al. 2015). Mindfulness is an important element in ACT protocols. In this conceptual framework, mindfulness is integrated as a practice that contributes to build the overarching behavioural pattern for psychological flexibility, which includes not only being open to internal 
and external events and being focused on the present moment, but also being committed to act in the direction of one's own values to adaptively build a meaningful life.

In this explorative single-arm open-label study, we aimed to assess whether an ACT-based training, conducted in a group of Italian children and adolescents with ADHD, could lead to changes in global functioning and behavioural symptoms, measurable through clinical rating scales specific for ADHD, while considering fundamental confounders.

\section{Methods}

\section{Participants}

All parents had completed an ACT-based Cognitive Behavioural Parent Training (according to the protocol described in Vanzin et al. 2018b) a maximum of 3 months before the evaluation of children for the inclusion in this study. Potential participants for this study were selected, following enrolment criteria, among all children with a diagnosis of ADHD enlisted for a group psychological treatment. A complete study flow-chart is provided in Fig. 1. A clinical sample of 36 children and adolescents who were referred to the Child Psychopathology Unit of our Institute for ADHD treatment was recruited over 3 years (from 2015 to 2018). Inclusion criteria were age between 8 and 13 years and a diagnosis of ADHD. All participants were diagnosed by a child neuropsychiatrist in accordance with the DSM 5 criteria (American Psychiatric Association [APA] 2013). A child psychologist experienced in the diagnosis of ADHD (LV) confirmed independently the diagnoses by through direct observation and the administration of the semistructured interview Development and Well-Being Assessment (DAWBA) (Goodman et al. 2000). The Wechsler Intelligence Scale for Children-III (Wechsler 1991) or -IV (Wechsler 2003) was used to obtain the Full Scale Intelligence Quotient (FSIQ). Only patients with FSIQ higher than 85 were included. Any major neurological or medical condition, a psychiatric diagnosis of autism spectrum disorder or specific language impairment (but no other psychiatric co-morbidities), or the fact that parents did not complete their ACT-based parent training, were considered exclusion criteria. Thirty-six children with ADHD were enrolled in the rehabilitation treatment program. Five dropped out of the study (14\%) and their baseline data were not analysed. Thirty-one patients remained in the study until its end and they had a complete set of data (Fig. 1).

Mean age was 10.7 years $(\mathrm{SD}=1.4)$ and the percentage of males was $93.5 \%(n=29)$. The mean socio-economic status (SES) of families was $59.5(\mathrm{DS}=19.5)$. Different types of ADHD were included in the sample: $80.6 \%$ of children were diagnosed Combined-ADHD, 9.7\%

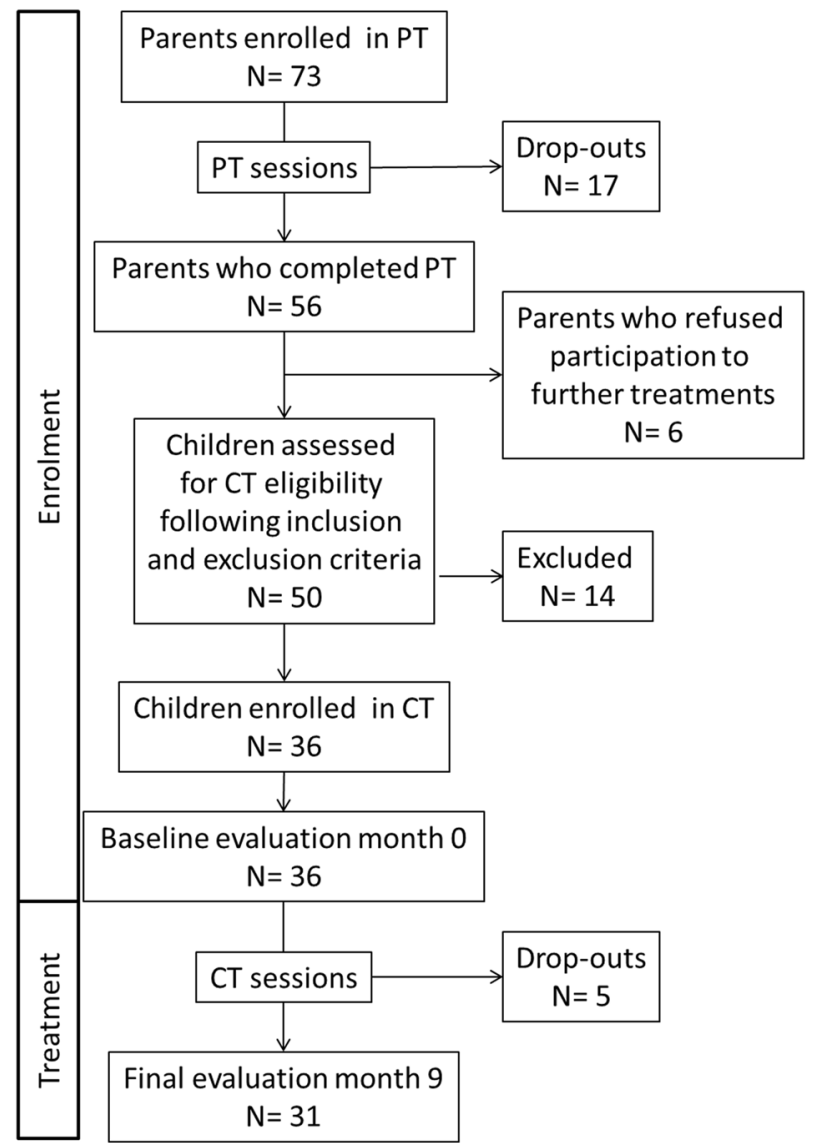

Fig. 1 Study flow-chart. This flow-chart describes the flow of parents and children through the initial parent-training course (not examined in this study) and the subsequent child training course. Parents of 73 children with ADHD were initially enrolled in the parent-training program; of these, the parents of 56 children completed the parenttraining and 50 agreed to enlist their children for child-training. Among them, 36 children met study criteria and received a baseline assessment, CT sessions, and a final assessment. For each family, less than 3 months passed between the completion of parent training and the baseline evaluation of children

predominantly Inattentive-ADHD, 6.5\% not otherwise specified and $3.2 \%$ predominantly Hyperactive-Impulsive ADHD. 16 patients (51.6\%) who received the treatment had a co-diagnosis with other psychopathologies, and among them $38.7 \%(n=12)$ of participants had a co-diagnosis with a learning disability or speech disorder. Patients' demographics and clinical characteristics are provided in Table 1.

\section{Procedure}

All subjects took part in a treatment program that lasted 9 months overall and consisted of 26 weekly group sessions of $90 \mathrm{~min}$. Each group was composed of four to five children. Before the intervention, therapists met with children and their parents to introduce the program, discuss potential benefits or shortcomings, investigate their motivation to 
Table 1 Clinical and demographic characteristics of the sample

\begin{tabular}{ll}
\hline & Sample $(N=31)$ \\
\hline Age at assessment (years) mean \pm SD & $10.7 \pm 1.4$ \\
Gender $N(\%)$ & \\
Female & $2(6.5 \%)$ \\
Male & $29(93.5 \%)$ \\
Full IQ mean \pm SD & $98.3 \pm 12.2$ \\
SES mean \pm SD & $59.5 \pm 19.5$ \\
Type of ADHD $(\%)$ & \\
Inattentive & $3(9.7 \%)$ \\
Hyperactive-Impulsive & $1(3.2 \%)$ \\
Combined & $25(80.6 \%)$ \\
NOS & $2(6.5 \%)$ \\
Co-diagnosis $(\%)$ & \\
NO & $15(48 \%)$ \\
YES & $16(52 \%)$ \\
Specific learning disorder & $12(38.7 \%)$ \\
Tic disorder & $3(9.7 \%)$ \\
Anxiety disorders & $2(6.5 \%)$ \\
Disruptive behaviour disorders & $1(3.2 \%)$ \\
Mood disorders & 0 \\
Pharmacological therapy $(\%)$ & \\
Methylphenidate & $3(9.7 \%)$ \\
None & $28(90.3 \%)$ \\
\hline
\end{tabular}

participate, introduce the reinforcement and punishment system and point out the importance of carrying out homework assignments and pursuing weekly objectives. The program aimed to gradually build the ability to contact and describe the own needs, wants and desires of patients and to reduce their problematic behaviour, while steering children and adolescents towards a more adaptive behavioural pattern. Experiential exercises helped to build sensitivity to actual contingencies in social situations, and mindfulness practice was used to help children notice thoughts, sensations, emotions and urges, and to accept them without reacting. Mindfulness training increased the ability of children to distance themselves from internal experiences and "kindly return" to awareness of the present. Moreover, treatment helped children to create a place to identify and choose what really matters for them in their lives. During the training, children developed not only mindfulness abilities, but also other psychological skills. They had the possibility to move through all the six core ACT processes: acceptance, cognitive defusion, being present, self as context, values and committed action. In sessions $1-4$, we created motivation to collaborate in a group context and introduced personal values and the mindfulness practice; we identified personal values in life domains and consistent "committed actions" as well as barriers (feelings, thoughts and external stimuli) to reaching personal goals. In session 5-14, we promoted defusion skills and the meaning of self as context, then we developed mindfulness abilities through specific exercises; we also taught children to read emotions as discriminative stimuli of an action compatible with the current context. In the fourth phase (sessions 16-19), we focused on perspective taking in order to foster empathy and social sensitivity. In sessions $20-22$, we reviewed and consolidated the contents learned in previous sessions, trying to help children to cope with problematic situations in their daily lives (inner or external) and enabling them to generalize learned skills. In the last phase (sessions 23 to 25 ), we re-elaborated in a creative way what the children had learnt (for example, making a movie to be seen with parents). The main aspect of treatment was the creation of a group metaphor that the trainers developed to suit children's language and experiences. The group metaphor creates an idea of shared interests, giving a sense of continuity to the training activity and supporting the collective ACT processes. For a short overview of the session themes and exercises, see Table 2.

We reinforced target behaviours during sessions and increased motivation to complete homework by using token economy. Our reinforcement schedule assigned a reinforcer if children displayed the target behaviour required for each activity. The target behaviour involved being present, engaged and aware of what was happening. After a conditioned signal sound, children could double their token gain if the whole group respected silence, took some deep breaths and listened to trainers. Treatment adherence and fidelity were rewarded using the token economy procedure, as described in the book by Vanzin (2018a). Through the token economy, we could increase children's interest and attendance to sessions and ensure the completion of homework. Patients earned points by complying to all these aspects, strongly increasing treatment adherence and fidelity. Sessions were conducted by two experienced psychotherapists among A.V., V.M., M.F. During the intervention, therapists met on a weekly basis to discuss group progress and monitor the state of treatment. Sessions were supervised weekly by an expert cognitive-behavioural psychotherapist (L.V.). In the context of this group training, children had one individual session after 4 months from treatment start. The individual sessions were supplemental face-to-face interviews between therapist and child, aimed to highlight the strengths and weaknesses of the child training, and to point out potential issues; in the context of these sessions, generalization of abilities acquired during the group training is promoted by analysing areas of daily life in which they could be applied. 
Table 2 Main therapeutic components and example of exercises for each block session

\begin{tabular}{|c|c|c|}
\hline Sessions number & Goals and therapeutic components & Main activities \\
\hline $1-4$ & $\begin{array}{l}\text { - Present the training program and increase } \\
\text { motivation to collaborate in a group context } \\
\text { - Introduce the group metaphor } \\
\text { - Induce 'creative hopelessness' } \\
\text { - Identify personal values in life domains and } \\
\text { committed actions } \\
\text { - Develop mindfulness abilities }\end{array}$ & $\begin{array}{l}\text { - Find out your personal values and committed actions } \\
\text { - Create your 'values compass' } \\
\text { - Mindfulness of breathing }\end{array}$ \\
\hline $5-9$ & $\begin{array}{l}\text { - Identify barriers (feelings, thoughts and external } \\
\text { stimulus) } \\
\text { - Increase awareness of thoughts } \\
\text { - Promote defusion skills } \\
\text { - Develop mindfulness abilities }\end{array}$ & $\begin{array}{l}\text { - Notice thoughts while performing an activity } \\
\text { - Dropping anchor exercises } \\
\text { - Mindfulness exercises: focus on the five senses }\end{array}$ \\
\hline $10-15$ & $\begin{array}{l}\text { - Increase awareness of emotions } \\
\text { - Use emotions as a discriminative stimulus of an } \\
\text { action compatible with the current context } \\
\text { - Develop mindfulness abilities }\end{array}$ & $\begin{array}{l}\text { - Watch cartoon scenes: describe triggers, emotions and behavioural } \\
\text { consequences } \\
\text { - Body scan exercise } \\
\text { - Role playing activities } \\
\text { - Draw my personal ‘values pathway' } \\
\text { - Mindfulness exercises: focus on the five senses }\end{array}$ \\
\hline $16-19$ & $\begin{array}{l}\text { - Train perspective taking } \\
\text { - Develop mindfulness abilities }\end{array}$ & $\begin{array}{l}\text { - Watch landscapes from different views } \\
\text { - Watch optical illusions } \\
\text { - Recognize what is clouding my sight } \\
\text { - Use perspective taking to understand social situations } \\
\text { - Mindfulness exercises }\end{array}$ \\
\hline $20-22$ & $\begin{array}{l}\text { - Train problem-solving } \\
\text { - Develop mindfulness abilities }\end{array}$ & $\begin{array}{l}\text { - ROAD model: R respira (breath), Osserva (observe), A ascolta i tuoi } \\
\text { valori (notice your values), D decidi come agire (choose your } \\
\text { actions) } \\
\text { - Find alternatives } \\
\text { - Mindfulness exercises }\end{array}$ \\
\hline $23-25$ & $\begin{array}{l}\text { - Re-elaborate in a creative way what the children } \\
\text { have learnt }\end{array}$ & - The group makes a video to be watched with their parents \\
\hline
\end{tabular}

This program was devised based on principles and methods from Vanzin (2018a)

\section{Measures}

Data on clinical conditions were collected, including pharmacological treatment, diagnosis and co-diagnoses and demographic characteristics including age, gender and socioeconomic status (SES). The Hollingshead 9-point scale for parental occupation (Hollingshead 1975) was used to rate SES on a scale from 10 to 90: subjects with scores between 10 and 30 had a low SES, subjects with scores between 40 and 60 had a medium SES, subject with scores between 70 and 90 had a high SES.

Child training effectiveness was measured through two outcome measures: Conners' Parent Rating Scales-Revised: Long version (CPRS-R:L) (Conners 2001) completed by the children's mothers and Clinical Global Impression-Severity scale (CGI-S) (Guy 1976) completed by non-blind independent clinicians. In the present study, CPRS-R:L and CGI$\mathrm{S}$ were administered before the beginning of treatment and after 9 months, i.e. at the end of treatment.

The CPRS-R:L is a reliable instrument for assessing ADHD and monitoring treatment (Pliszka et al. 2007). CPRS-R:L is a questionnaire used to assesses behavioural problems and ADHD symptoms, to be completed by parents. The CPRS-R:L, suitable for children aged 3 to 17, provides an assessment of behavioural and psychopathological problems and it particularly targets ADHD symptoms. The Italian version adapted by Nobile et al. (2007) is an 80item scale that produces $\mathrm{T}$-scores for 14 subscales: cognitive problems/inattention, oppositional behaviour, hyperactivity, anxiety and shyness, perfectionism, social problems, psychosomatic behaviour, CPRS-R:L Global Index (comprising Restless-Impulsive and Emotional Lability), ADHD Index and DSM-IV symptoms subscales (comprising DSM-IV Inattentive and DSM-IV HyperactiveImpulsive). The CPRS-R:L has well-established reliability and validity (Conners 2001) in rating the behaviour of children with ADHD and in assessing an intervention's effectiveness. In the present sample, before treatment, the oppositional behaviour, cognitive problems/inattention, hyperactivity, social problems, psychosomatic behaviour, CPRS-R:L Global Index, ADHD Index and DSM-IV symptoms subscales showed an acceptable to good internal reliability (Cronbach's $\alpha$ ranging from 0.766 to 0.853 ); anxiety and shyness and perfectionism subscales showed a 
questionable reliability (Cronbach's $\alpha 0.680$ and 0.657 , respectively). After treatment, the oppositional behaviour, cognitive problems/inattention, hyperactivity, psychosomatic behaviour, CPRS-R:L Global Index, ADHD Index and DSM-IV symptoms subscales showed an acceptable to excellent internal reliability (Cronbach's $\alpha$ ranging from 0.711 to 0.915 ); social problems, anxiety and shyness and perfectionism subscales showed a questionable reliability (Cronbach's $\alpha$ 0.668, 0.655 and 0.273, respectively).

The CGI-S provides an overall clinician-determined summary of psychopathologic severity on a 7-point scale ( $1=$ normal; $7=$ extremely ill) (Busner and Targum 2007) and is one of the most widely used assessment tools in psychiatry.

Both assessment tools have demonstrated acceptable reliability and validity in previous studies with Italian children (Masi et al. 2013; Masi et al. 2014). Since this was a preliminary study, we applied no blinding. However, the CGI-S rater was a psychiatrist independent from the treating clinical psychologists.

\section{Data Analysis}

Descriptive statistics were performed for age, gender, SES, type of ADHD and co-diagnosis. Normality tests were used to determine if variables and scores of each CPRS-R:L subscale had a normal distribution. Mean and standard deviation (SD) were used for normally distributed continuous variables, median and interquartile range for nonnormally distributed variables, while frequency and percentage were used for categorical variables. In order to determine if CPRS-R:L subscale scores were significantly different before vs. after treatment, we performed paired samples T-tests or Wilcoxon signed rank tests, as required. The significance level was set at $p<0.05$ and effect size was expressed as Cohen's $d$ or Wilcoxon's $r$, as required. We aimed to control these analyses for possible confounding variables such as medication (The MTA Cooperative Group, 1999), social economic status (Nobile et al. 2010, Biederman et al. 2002) and comorbidity (Frigerio et al. 2006, Bianchi et al. 2017), which were found to be associated with severity of symptoms and different response to treatment. Gender and age were not included in our list of covariates, because we analysed CPRS T-scores, which are already weighed for age and gender. We used repeated measures ANCOVA models to assess before vs. after treatment changes controlled for covariates: treatment was used as the within-group factor; pharmacotherapy $x$ comorbidity $\times$ SES as between-group factor. We applied the model to each CPRS-R:L subscale that was significantly changed in non-controlled analyses. We reported the results of ANCOVAs for each CPRS-R:L subscale, including the significance and $R^{2}$ of the whole model, and the significance and effect size (ES) as partial eta squared for treatment, to check if it was still significant after controlling for covariates. Changes on the CGI-S scale were assessed by the Wilcoxon Signed Rank Test. All analyses were conducted using IBM SPSS Statistics, version 22.0 SPSS.

\section{Results}

Significant differences between scores measured before vs. after treatment were found in the subscales Oppositional $(p=0.048, d=0.31)$ Cognitive Problems $(p=0.005, d=$ $0.5)$, Hyperactivity $(p=0.006, d=0.56)$, Perfectionism $(p=0.017, r=0.3)$, ADHD Index $(p=0.023, d=0.4)$, Global Index: Restless - Impulsive $(p=0.023, d=0.44)$, Global Index: Total $(p=0.036, d=0.39)$, DSM IV Inattentive $(p=0.029, d=0.45)$, DSM IV Hyperactive Impulsive $(p=0.016, r=0.31)$, DSM IV Total $(p=0.003$, $d=0.57$ ). The complete list of results is available in Table 3.

We re-analysed changes on the CPRS scores by ANCOVA, taking into account the potential influence of covariates including concomitant drug treatment, presence of comorbidities and SES. As shown in Table 4, the effect of treatment remained significant for the CPRS subscales Perfectionism (partial $\eta^{2}=0.31, p<0.01$ ), Global Index: Restless-Impulsive (partial $\eta^{2}=0.29, p<0.01$ ), Global Index: Total (partial $\eta^{2}=0.31, \quad p<0.01$ ), DSM IV Hyperactive-Impulsive (partial $\eta^{2}=0.20, p=0.02$ ).

CGI-S scores demonstrated global improvement: before treatment the CGI-S median was 4 (interquartile range 1), and 3 after treatment. This change was significant $(p<0.001$ at Wilcoxon Signed Rank Test). After treatment, $74.2 \%$ of the patients $(n=23)$ improved in the severity index. Of this, $19(61.3 \%)$ patients shifted one step, and $4(13 \%)$ more than one step. No patient presented with a worse CGI-S score after treatment. Details are presented in Table 5.

\section{Discussion}

The aim of the present study was to explore the efficacy of an ACT-based treatment, in adjunct to token economy and previous parent training, in reducing impulsiveness and improving self-regulation in children with ADHD. This study of a 'third wave' behavioural treatment in a groupbased setting is pivotal, as it examined measurable changes in ADHD rating scales (CPRS-R:L and CGI-S) and not only in ACT processes; the positive results we observed support a real therapeutic efficacy of this ACT-based child training. During the training, children had the possibility to move through the six ACT processes: they were exposed to the contact with the present moment, to the process of 
Table 3 Comparison of CPRS scores before and after treatment

\begin{tabular}{lccll}
\hline CPRS subscales & Before & After & $p$-value & Effect size $(d$ or $r)$ \\
\hline Oppositional & $65.4(12.3)$ & $61.3(14.0)$ & 0.048 & 0.31 \\
Cognitive problems & $71.4(11.4)$ & $66.1(9.3)$ & 0.005 & 0.50 \\
Hyperactivity & $69.7(10.9)$ & $63.6(11.0)$ & 0.006 & 0.56 \\
Anxious-Shy & $47.8(8.8)$ & $46.9(9.1)$ & 0.495 & 0.10 \\
Perfectionism $^{\text {a }}$ & $49(13)$ & $47(10)$ & 0.017 & 0.30 \\
Social problems $^{\text {a }}$ & $61(29)$ & $55(17)$ & 0.338 & 0.12 \\
Psychosomatic $^{\text {a }}$ & $41(21)$ & $47(16)$ & 0.134 & 0.19 \\
ADHD Index $^{\text {Global Index: Restless-Impulsive }}$ & $70.77(9.9)$ & $66.29(10.2)$ & 0.023 & 0.44 \\
Global Index: Emotional Lability $^{\mathrm{a}}$ & $61(13)$ & $54(25)$ & 0.360 & 0.12 \\
Global Index: Total $^{\text {DSM IV Inattentive }}$ & $69.81(11.2)$ & $65.35(11.2)$ & 0.036 & 0.39 \\
DSM IV Hyperactive-Impulsive $^{\mathrm{a}}$ & $71.94(10.8)$ & $67.26(9.8)$ & 0.029 & 0.45 \\
DSM IV Total $^{71(12)}$ & $63(16)$ & 0.016 & 0.31 \\
\hline
\end{tabular}

Results are presented either as: (a) means with standard deviations, followed by paired-samples $t$-tests and Cohen's $d$ for normally distributed variables; or (b) medians with interquartile ranges, followed by pairedsamples Wilcoxon signed rank tests and Wilcoxon's $r$ for non-normally distributed variables

andicates non-normally distributed variables
Table 4 Statistical relevance of covariates for CPRS score changes

\begin{tabular}{|c|c|c|c|c|c|c|}
\hline \multirow[t]{2}{*}{ CPRS subscale } & \multicolumn{3}{|c|}{ Full model } & \multicolumn{3}{|c|}{$\begin{array}{l}\text { Treatment } \\
\text { component }\end{array}$} \\
\hline & $F$ & $p$ & $R^{2}$ & $F$ & $p$ & ES \\
\hline Oppositional & 10.2 & 0.01 & 0.97 & 1.0 & 0.34 & 0.04 \\
\hline Cognitive problems & 2.0 & 0.15 & 0.08 & 1.6 & 0.22 & 0.06 \\
\hline Hyperactivity & 6.5 & $<0.01$ & 0.32 & 1.8 & 0.19 & 0.07 \\
\hline Perfectionism & 3.3 & 0.08 & 0.11 & 11.9 & $<0.01$ & 0.31 \\
\hline ADHD Index & 10.2 & $<0.01$ & 0.28 & 2.4 & 0.14 & 0.08 \\
\hline $\begin{array}{l}\text { Global Index: } \\
\text { Restless-Impulsive }\end{array}$ & 6.5 & 0.02 & 0.20 & 10.4 & $<0.01$ & 0.29 \\
\hline Global Index: Total & 13.8 & $<0.01$ & 0.35 & 11.5 & $<0.01$ & 0.31 \\
\hline DSM IV Inattentive & 5.8 & 0.02 & 0.18 & 2.2 & 0.15 & 0.08 \\
\hline $\begin{array}{l}\text { DSM IV } \\
\text { Hyperactive-Impulsive }\end{array}$ & 11.7 & $<0.01$ & 0.31 & 6.5 & 0.02 & 0.20 \\
\hline DSM IV Total & 10.4 & $<0.01$ & 0.29 & 3.9 & 0.06 & 0.13 \\
\hline
\end{tabular}

The full model comprises Treatment (within-patient factor, comprising: the whole clinical treatment, ACT, token economy, delayed effects of parent training; passing of time; other unpredictable pre-post effects) $\times($ Pharmacotherapy $\times$ Comorbidity $\times$ SES) (between-patients factor). Effect-sizes (ES) are shown as partial eta-squared

acceptance, to the cognitive defusion, to the self as context, to values and to committed actions. Children gradually built the ability to contact and describe their own needs, wants and desires. Through the experiential exercises, the analysis of social situations and enhancement of perspective taking abilities, we led children into using a contingency-governed approach for choosing their behaviours.
The clinical question addressed in this study concerned the effectiveness of ACT-based child training for ADHD rehabilitation. Although we observed a quite small sample of children, we controlled the results for the potential effect of confounding variables such as pharmacotherapy, the presence/absence of comorbidity and SES. We found several cases in which pharmacotherapy, comorbidity and SES may have significantly influenced the outcome of therapy. A quantitative examination of the role of these covariates may deserve further investigation in larger and more representative studies. In particular, after controlling for these confounders, the effect of treatment remained significant, and retained a medium effect size, regarding the four following CPRS subscales. Changes in the subscale Perfectionism, although significant, were minimal and scores were always below the clinical attention threshold. Improvements in the subscales Global Index: Restless-Impulsive, Global Index: Total and DSM IV Hyperactive-Impulsive suggest that treatment may have had an effect on impulsiveness and, indirectly, on emotional aspects. The reduction of impulsive acts, observed by parents, might be interpreted as a consequence of the mindfulness components of the present treatment. Children may have learned to observe emotions and thoughts, without reacting impulsively. Indeed, mindfulness consists also of observing thoughts and emotions and distancing from them, which reduces impulsive and automatic reactions (Peters et al. 2015). By freeing themselves from the cycle of thinking-feeling-acting, children may acquire the space necessary to concentrate on what they are doing in the present moment, to reflect, and to learn. Another hypothesis 
Table 5 Observed shifts in individual CGI-S scores before and after treatment

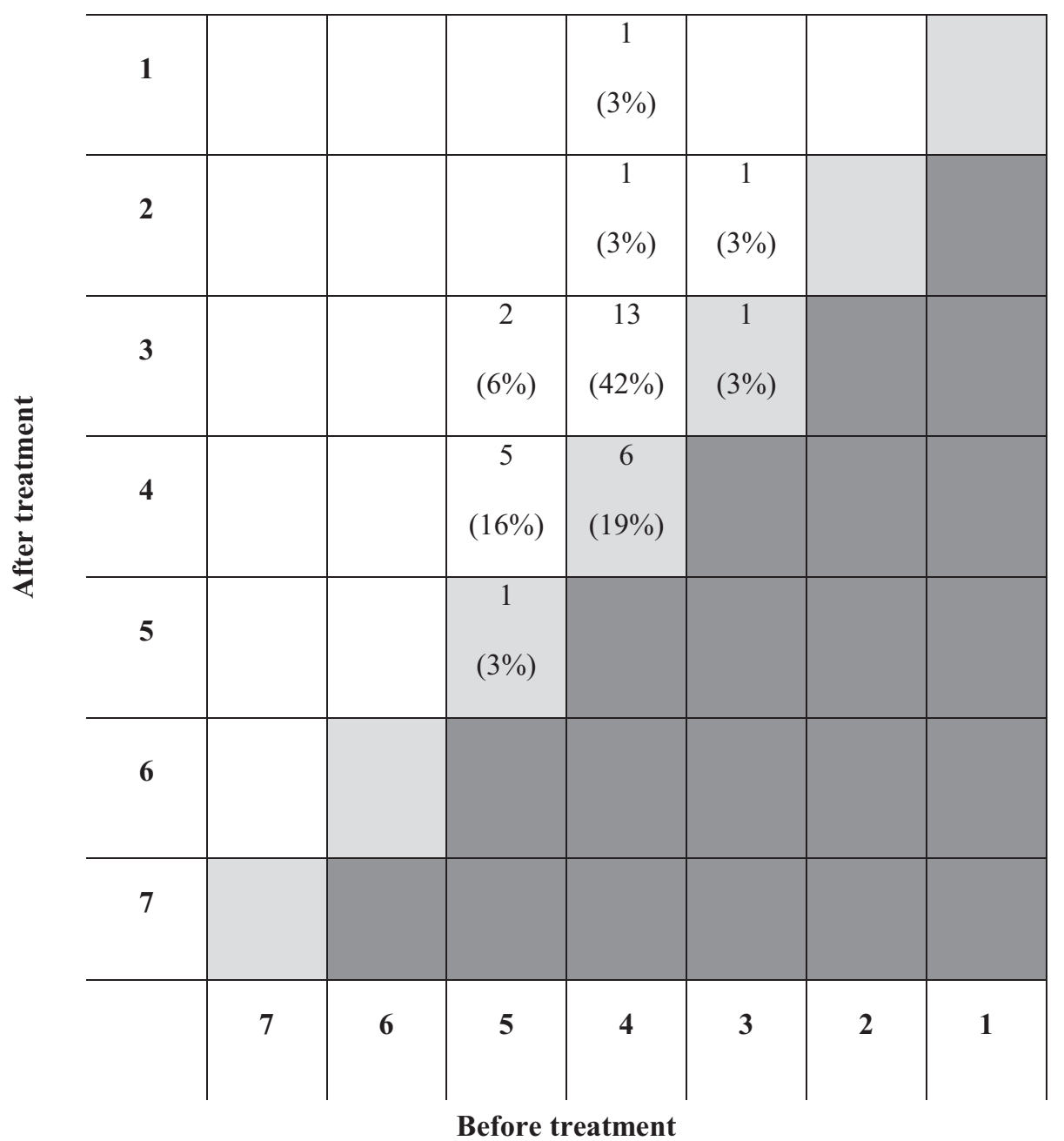

Numbers and percentages refer to individual patients. Dark grey cells indicate patients who shifted to a higher CGI-S category after treatment. Light grey cells indicate patients who remained in their baseline CGI-S category also after treatment. White cells indicate patients who shifted to a lower CGI-S category

CGI-S Clinical Global Impressions-Severity: $1=$ normal; $2=$ borderline; $3=$ mildly ill; $4=$ moderately ill; $5=$ markedly ill; $6=$ severely ill; $7=$ extremely ill

is that children may have improved their level of awareness of mind-wandering, which is the first step required to allow attention to be re-focused on a specific task. Results also demonstrated a reduction in general psychopathology (CPRS Global Index Total). The CPRS Global Index Total is informative regarding changes in both externalised symptoms and emotional or internalised symptoms and thus it is helpful for monitoring treatment progress (Conners 2001). Therefore, the results suggest the possibility of conducting an ACT-based child training in this population. It is possible to speculate that the effect size of treatment may be higher than the one we observed in this preliminary study, due to the small sample size, and most of all due to the fact that this ACT-based CT still has to be optimized.
Since this is an exploratory study, we also considered non-controlled results. The largest improvements over time were in the CPRS-R:L subscales Cognitive Problems, Hyperactivity, DSM IV Inattentive and DSM IV Total, as shown by effect size. The Cognitive Problems and DSM IV Inattentive subscales refer to the possibility that children may struggle to concentrate, may be easily distracted by external stimuli, may not pay attention to details, may make frequent mistakes from distraction, may be 'forgetful' and may have organisational problems and difficulties in completing tasks. The fact that two CPRS-R:L subscales consistently indicated a reduction of inattention allows us to speculate that practicing mindfulness may have fostered in children a higher awareness of moments of distraction and a 
better ability to return with their minds to the 'here and now'. In fact, an important part of this child training course consisted of leading children into developing awareness of their own thoughts, thus becoming able to detect distractions. Children with ADHD are continuously shifting their attention focus and are not aware of being distracted. We believe that they should be encouraged to improve their awareness of distractions before enhancing their ability to maintain attention. The Hyperactivity subscale refers to difficulties in sitting or dedicating themselves to the same task for a long time, as well as restlessness. Our hypothesis is that work on the acceptance of one's emotions and impulses, whatever they may be, may have helped the children to accept the presence of a minor discomfort (the desire to move or to leave the activity to start another one) when this was necessary to achieve something important for them. This ACT process is described by the term 'selfregulation'. During the training, children were encouraged to explore and reflect on what is important in daily life activities, sharing with the rest of the group personal qualities that they considered important to show. We had the opportunity to guide children into choosing functional behaviours to develop their qualities, while experiencing the possibility of not acting immediately on every impulse or every thought that crossed their mind.

Through CGI-S scores, we assessed the severity of patients' illness and found a significant improvement after treatment. In our sample, $74.2 \%$ of the children shifted from a more severe functional impairment class to a less severe one. Therefore, there is agreement between clinicians and parents regarding the patients' general improvement.

\section{Limitations and Future Research Directions}

Results of the present work must be considered preliminary, as a control group would be necessary to assess treatment efficacy properly. For the same reason, we did not perform corrections for multiple comparisons. Another limitation was in the sample size of 31 patients, which was still a larger cohort as compared to previous studies. Although it would be best to include fewer covariates in the ANCOVA with a sample size of 31 , we included pharmacotherapy, comorbidities and SES, as they are all fundamental from a clinical point of view. Our study did not comprise an evaluation of patients who dropped out of treatment: the most/ least severe participants may have dropped out of treatment, thus biasing results. In addition, our current working protocol does not involve a follow-up reassessment of outcomes, which is a crucial aspect to verify the generalization of treatment achievements. Regarding the generalizability of the present study, our sample is in line with the Italian population (Bonati et al. 2015), although we acknowledge that the epidemiology of ADHD in Italy is not overlapping with that of other countries, and this constitutes a limitation of our study. The ACT-based cognitive behavioural parent training was completed before the ACT-based cognitive behavioural child training started. Therefore, our baseline evaluation took into account the immediate effects of parent training. However, the parent training may have had ongoing effects that cannot be accounted for and that may have contributed to the treatment effects in the present study. Another aspect we could not control for, was the possibility of a direct effect of the token economy system. Unfortunately, the lack of a control group did not allow us to investigate these aspects, which constitutes a limitation of the present study. We evaluated the intervention as a package, although some specific components of the program were evidence-based for ADHD (e.g.: token systems) and others were not (e.g.: mindfulness practice). Further research is needed to single out the effective and ineffective components of the program and improve its efficiency. In order to assess the importance of the token economy within the ACT context, future studies should include a waiting list group, a CBT CT group and an ACT CT group, with both training groups including the token economy. Our study relied on parents' reports of symptoms and ratings of nonblind clinicians; thus, findings may have been influenced by expectancy effects. We recommend for the future to include direct measures of attention and impulsive behaviour, perhaps through neuropsychological tasks. Moreover, investigating the generalization of changes, for instance to the school setting, was not within the aims of this work and it should be investigated in future studies.

Our results indicate an improvement in line with the only previous study on the feasibility of ACT with ADHD children, which investigated changes in ACT processes (Murrel et al. 2015). This preliminary study demonstrates the effectiveness of ACT-based child training in a sample of children with ADHD using clinical rating scales specific for ADHD and considering fundamental confounders such as pharmacotherapy, comorbidity and SES. The promising results of this study should encourage the conduction of future clinical trials on the effectiveness of ACT-based child training for children with ADHD, possibly including a larger number of patients with more homogeneous characteristics. Such studies should also apply clinical rating scales including the CPRS-R:L and CGI, in order to provide a critical mass of evidence that might be used in the future to guide innovative clinical interventions based on the ACT approach.

Acknowledgements The financial support from the Italian Ministry of Health (Ricerca Corrente 2019, to MP, MN, MM) is gratefully acknowledged. The funding public institutions had no role in any part of the work. 
Authors' Contribution LV conceived the study and wrote the manuscript; AV and VM collected data and wrote the manuscript; MP analysed data and critically supervised manuscript writing; AO, AR analysed data; GP, MM conceived the study; MN conceived the study and critically supervised manuscript writing.

\section{Compliance with Ethical Standards}

Conflict of Interest The authors declare that they have no conflict of interest.

Ethical Approval This study received approval by the Ethics Committee of the Scientific Institute I.R.C.C.S. E. Medea, scientific section of the Association "La Nostra Famiglia", Bosisio Parini (LC), Italy. The study was performed in accordance with the ethical standards set forth in the 1964 Declaration of Helsinki and its later amendments.

Informed Consent Written informed consent and assent was proposed to all parents and participants, respectively. Only in case they both agreed to participate, patients were enrolled in this study.

Publisher's note Springer Nature remains neutral with regard to jurisdictional claims in published maps and institutional affiliations.

Open Access This article is distributed under the terms of the Creative Commons Attribution 4.0 International License (http://crea tivecommons.org/licenses/by/4.0/), which permits unrestricted use, distribution, and reproduction in any medium, provided you give appropriate credit to the original author(s) and the source, provide a link to the Creative Commons license, and indicate if changes were made.

\section{References}

American Psychiatric Association. (2013). Diagnostic and statistical manual of mental disorders (5th ed.). Arlington, VA: American Psychiatric Publishing.

A-Tjak, J. G. L., Davis, M. L., Morina, N., Powers, M. B., Smits, J. A. J., \& Emmelkamp, P. M. G. (2015). A meta-analysis of the efficacy of acceptance and commitment therapy for clinically relevant mental and physical health problems. Psychotherapy and Psychosomatics, 84(30), 30-36.

Barkley, R. A. (1997). Attention-deficit/hyperactivity disorder, selfregulation, and time: toward a more comprehensive theory. Journal of Developmental \& Behavioral Pediatrics, 18(4), 271-279.

Barkley, R. A., Murphy, K. R. \& Fischer, M. (Eds) (2008). ADHD in adults: what the science says. New York, NY: The Guilford Press.

Bianchi, V., Brambilla, P., Garzitto, M., Colombo, P., Fornasari, L., Bellina, M., \& Perna, G. (2017). Latent classes of emotional and behavioural problems in epidemiological and referred samples and their relations to DSM-IV diagnoses. European Child \& Adolescent Psychiatry, 26(5), 549-557.

Bianchini, R., Postorino, V., Grasso, R., Santoro, B., Migliore, S., Burlò, C., \& Mazzone, L. (2013). Prevalence of ADHD in a sample of Italian students: a population-based study. Research in Developmental Disabilities, 34(9), 2543-2550.

Biederman, J., Mick, E., Faraone, S. V., Braaten, E., Doyle, A., Spencer, T., \& Johnson, M. A. (2002). Influence of gender on attention deficit hyperactivity disorder in children referred to a psychiatric clinic. American Journal of Psychiatry, 159(1), $36-42$.
Bonati, M., Reale, L., Zanetti, M., Cartabia, M., Fortinguerra, F., Capovilla, G., \& Martinelli, O. (2015). A regional ADHD centerbased network project for the diagnosis and treatment of children and adolescents with ADHD. Journal of Attention Disorders, 22 (12), 1173-1184.

Burke, C. A. (2010). Mindfulness-based approaches with children and adolescents: a preliminary review of current research in an emergent Field. Journal of Child and Family Studies, 19(2), 133-144.

Busner, J., \& Targum, S. D. (2007). The clinical global impressions scale: applying a research tool in clinical practice. Psychiatry, 4 (7), 28-37.

Castellanos, F. X., \& Tannock, R. (2002). Neuroscience of attentiondeficit/hyperactivity disorder: the search for endophenotypes. Nature Reviews Neuroscience, 3(8), 617.

Chambles, D. L., \& Ollendick, T. H. (2001). Empirically supported psychosocial interventions: Controversies and evidence. Annual Reviews of Psychology, 52, 685-716.

Ciarrochi, J., Hayes, L. \& Bailey, A. (Eds) (2012). Get out of your mind and into your life for teens. Oakland, CA: New Harbinger.

Conners, C. K. (2001). Conners' rating scales-revised (Technical Manual). North Tonawanda, NY: Multi-Health Systems, Inc.

Donfrancesco, R., Marano, A., Calderoni, D., Mugnaini, D., Thomas, F., Di Trani, M., \& Vitiello, B. (2014). Prevalence of severe ADHD: an epidemiological study in the Italian regions of Tuscany and Latium. Epidemiology and Psychiatric Sciences, 24(6), 525-533.

Frigerio, A., Vanzin, L., Pastore, V., Nobile, M., Giorda, R., Marino, C., \& Lenti, C. (2006). The Italian preadolescent mental health project (PrISMA): rationale and methods. International Journal of Methods in Psychiatric Research, 15(1), 22-35.

Gallucci, F., Berardi, C., Gallai, V., Pfanner, P., \& Weinberg, A. (1993). Symptoms of attention-deficit hyperactivity disorder in an Italian school sample: findings of a pilot study. Journal of the American Academy of Child and Adolescent Psychiatry, 32(5), 1051-1058.

Goodman, R., Ford, T., Richards, H., Gatward, R., \& Meltzer, H. (2000). The development and well being assessment: description and initial validation of an integrated assessment of child and adolescent psychopathology. Journal of Child Psychology and Psychiatry, 41(5), 645-655.

Greco, L. A., \& Hayes, S. C. (Eds). (2008). Acceptance and mindfulness treatments for children and adolescents: A practitioner's guide. Oakland, CA: New Harbinger.

Guy, W. (1976). ECDEU assessment manual for psychopharmacology. Rockville, MD: National Institute for Mental Health.

Hayes, S. C., Strosahl, K. D. \& Wilson, K. G. (Eds) (1999). Acceptance and commitment therapy: an experiential approach to behavior change. New York, NY: The Guilford Press.

Hayes, S. C., Barnes-Holmes, D., \& Roche, B. (Eds). (2001). Relational frame theory: a post-Skinnerian account of human language and cognition. New York, NY: Kluwer/Plenum.

Hayes, S. C., Follette, V. M., \& Linehan, M. M. (Eds). (2004). Mindfulness and acceptance: expanding the cognitive-behavioral tradition. New York, NY: The Guilford Press.

Hayes, S. C., Luoma, J. B., Bond, F. W., Masuda, A., \& Lillis, J. (2006). Acceptance and commitment therapy: model, processes and outcomes. Behaviour Research and Therapy, 44(1), 1-25.

Hollingshead, A. A. (1975). Four-factor index of social status. Yale University, New Haven, CT: unpublished manuscript.

Kabat-Zinn, J. (1994). Wherever you go, there you are: mindfulness meditation in everyday life. New York, NY: Hyperion. p.4.

Masi, G., Muratori, P., Manfredi, A., Lenzi, F., Polidori, L., Ruglioni, L., \& Milone, A. (2013). Response to treatments in youth with disruptive behavior disorders. Comprehensive Psychiatry, 54(7), 1009-1015. 
Masi, G., Milone, A., Paciello, M., Lenzi, F., Muratori, P., Manfredi, A., \& Muratori, F. (2014). Efficacy of a multimodal treatment for disruptive behavior disorders in children and adolescents: focus on internalizing problems. Psychiatry Research, 219(3), 617-624.

Matheson, L., Asherson, P., Wong, I. C. K., Hodgkins, P., Setyawan, J., Sasane, R., \& Clifford, S. (2013). Adult ADHD patient experiences of impairment, service provision and clinical management in England: a qualitative study. BMC Health Services Research, 13(1), 184.

The MTA Cooperative Group. (1999). A 14-month randomized clinical trial of treatment strategies for attention-deficit/hyperactivity disorder. Multimodal treatment study of children with ADHD. Archives of General Psychiatry, 56(12), 1073-1086.

Mugnaini, D., Masi, G., Brovedani, P., Chelazzi, C., Matas, M., Romagnoli, C., \& Zuddas, A. (2006). Teacher reports of ADHD symptoms in Italian children at the end of first grade. European Psychiatry, 21(6), 419-426.

Murrell, A. R., Steinberg, D. S., Connally, M. L., Hulsey, T., \& Hogan, E. (2015). Acting out to ACTing on: a preliminary investigation in youth with ADHD and co-morbid disorders. Journal of Child and Family Studies, 24(7), 2174-2181.

Nobile, M., Alberti, B. \& Zuddas, A. (Eds) (2007). Conners' rating scales-revise. Manuale [Conners' rating scales-revised, Manual]. Firenze, Italy: Giunti O.S.

Nobile, M., Rusconi, M., Bellina, M., Marino, C., Giorda, R., Carlet, O., \& Battaglia, M. (2010). COMT Val158Met polymorphism and socioeconomic status interact to predict attention deficit/ hyperactivity problems in children aged 10-14. European Child and Adolescent Psychiatry, 19(7), 549-557.

Öst, L. G. (2014). The efficacy of acceptance and commitment therapy: an updated systematic review and meta-analysis. Behaviour Research and Therapy, 61, 105-121.

Pelham, W. E., \& Fabiano, G. A. (2008). Evidence-based psychosocial treatments for Attention-Deficit/Hyperactivity Disorder. Journal of Clinical Child and Adolescent Psychology, 37(1), 184-214.

Peters, J. R., Smart, L. M., Eisenlohr-Moul, T. A., Geiger, P. J., Smith, G. T., \& Baer, R. A. (2015). Anger rumination as a mediator of the relationship between mindfulness and aggression: the utility of a multidimensional mindfulness model. Journal of Clinical Psychology, 71(9), 871-884.

Pliszka, S., American Academy of Child \& Adolescent Psychiatry Work Group on Quality Issues. (2007). Practice parameter for the assessment and treatment of children and adolescents with attention-deficit/hyperactivity disorder. Journal of the American Academy of Child and Adolescent Psychiatry, 46(7), 894-921.

Powers, M. B., Zum Vorde Sive Vording, M. B., \& Emmelkamp, M. P. (2009). Acceptance and Commitment Therapy: a meta-analytic review. Psychotherapy and Psychosomatics, 78(2), 73-80.

Puiu, A. A., Wudarczyk, O., Goerlich, K. S., Votinov, M., Konrad, K., Turetsky, B., \& Herpertz-Dahlmann, B. (2018). Impulsive aggression and response inhibition in attention-deficit/hyperactivity disorder and disruptive behavioral disorders: findings from a systematic review. Neuroscience \& Biobehavioral Reviews, 90, 231-246.

Ruiz, F. J. (2010). A review of acceptance and commitment therapy (ACT) empirical evidence: correlational, experimental psychopathology, component and outcome studies. International Journal of Psychology and Psychological Therapy, 10(1), 125-162.

Sagvolden, T., Russell, V. A., Aase, H., Johansen, E. B., \& Farshbaf, M. (2005). Rodent models of attention deficit/hyperactivity disorder. Biological Psychiatry, 57(11), 1239-1247.

Semple, R. J., Reid, E. F. G., \& Miller, L. F. (2005). Treating anxiety with mindfulness: an open trial of mindfulness training for anxious children. Journal of Cognitive Psychotherapy: An International Quarterly, 19(4), 379-392.

Semple, R. J., Lee, J., Rosa, D., \& Miller, L. F. (2010). A randomized trial of mindfulness based cognitive therapy for children: promoting mindful attention to enhance social-emotional resiliency in children. Journal of Child and Family Studies, 19(2), 218-229.

Shapiro, S. L., Carlson, L. E., Astin, J. A., \& Freedman, B. (2006). Mechanisms of mindfulness. Journal of Clinical Psychology, 62 (3), 373-386.

Sharma, A., \& Couture, J. (2014). A review of the pathophysiology, etiology, and treatment of attention-deficit hyperactivity disorder (ADHD). Annals of Pharmacotherapy, 48(2), 209-225.

Shiels, K., \& Hawk Jr, L. W. (2010). Self-regulation in ADHD: the role of error processing. Clinical Psychology Review, 30(8), 951-961.

Simons, J. S., \& Gaher, R. M. (2005). The Distress Tolerance Scale: development and validation of a self-report measure. Motivation and Emotion, 29(2), 83-102.

Stein, M. A. (2008). Impairment associated with adult ADHD. CNS Spectrums, 13(S12), 9-11.

Swain, J., Hancock, K., Dixon, A., \& Bowman, J. (2015). Acceptance and commitment therapy for children: a systematic review of intervention studies. Journal of Contextual Behavioral Science, 4 (2), 73-85.

Teasdale, J. D., Segal, Z. V., Williams, J. M. G., \& Mark, G. (1995). How does cognitive therapy prevent depressive relapse and why should attentional control (mindfulness) training help? Behavior Research and Therapy, 33(1), 25-39.

Thomas, R., Sanders, S., Doust, J., Beller, E., \& Glasziou, P. (2015). Prevalence of attention-deficit/hyperactivity disorder: a systematic review and meta-analysis. Pediatrics, 135(4), e994-e1001.

Van de Weijer-Bergsma, E., Formsma, A. R., De Bruin, E. I., \& Bögels, S. M. (2012). The effectiveness of mindfulness training on behavioral problems and attentional functioning in adolescents with ADHD. Journal of Child and Family Studies, 21(5), 775-787.

Van der Oord, S., Bögels, S. M., \& Peijnenburg, D. (2012). The effectiveness of mindfulness training for children with ADHD and mindful parenting for their parents. Journal of Child and Family Studies, 21(1), 139-147.

Vanzin, L. (2018a). Rallentiamo...e scegliamo! Child Trainng per l'ADHD [Slow down... and choose! Child Training for ADHD]. Milano, Italy: FrancoAngeli.

Vanzin, L., Colombo, P., Valli, A., Mauri, V., Ceccarelli, S. B., Pozzi, M., \& Nobile, M. (2018b). The effectiveness of coping power program for ADHD: an observational outcome study. Journal of Child and Family Studies, 27(11), 3554-3563.

Wechsler, D. (1991). Wechsler Intelligence Scale for Children - Third Edition. San Antonio, TX: The Psychological Corporation.

Wechsler, D. (2003). Wechsler Intelligence Scale for Children-Fourth Edition. San Antonio, TX: Harcourt Assessment, Inc.

Wilson, K. G., \& Luciano, C. (Eds). (2002). Terapia de aceptación y compromiso. Un tratamiento conductual orientado a los valores [Acceptance and Commitment Therapy (ACT): A Value-Oriented Behavioral Treatment]. Madrid, Spain: Editorial Pirámide.

Zuddas, A., Marzocchi, G. M., Oosterlaan, J., Cavolina, P., Ancilletta, B., \& Sergeant, J. (2006). Factor structure and cultural factors of disruptive behaviour disorders symptoms in Italian children. European Psychiatry, 21(6), 410-418.

Zylowska, L., Ackerman, D. L., Yang, M. H., Futrell, J. L., Horton, N. L., Hale, T. S., \& Smalley, S. L. (2008). Mindfulness meditation training in adults and adolescents with ADHD: a feasibility study. Journal of Attention Disorders, 11(6), 737-746. 